\title{
Perfil clínico de pacientes con sospecha de apneas del sueño
}

\author{
Clinical profile of patients suspected of sleep apnea
}

Perfil clínico de pacientes com suspeita de apneia do sono

Ramiro Horacio Rojas Mendiola ${ }^{1,3}$, Marcela Smurra ${ }^{1}$, Marina Khoury².

La apnea del sueño es el trastorno respiratorio del sueño más frecuente y afecta entre el 4 y el $20 \%$ de la población general. Es una enfermedad que se caracteriza por pausas en la respiración durante el sueño que se repiten durante toda la noche causando una inadecuada oxigenaci'ón de la sangre y una mala calidad de sueño. Los síntomas como el ronquido y la tendencia a quedar dormido durante el día muchas veces son minimizados por los pacientes y desestimados por profesionales de la salud. Esta enfermedad sin tratamiento lleva a complicaciones de salud, principalmente cardiovasculares; así como también a problemas laborales, de aprendizaje y a un riesgo aumentado de accidentes de tránsito. Por estos motivos es importante conocer sus síntomas y signos para diagnosticarla a tiempo y tratarla adecuadamente.

\section{Conceptos clave}

Qué se sabe sobre el tema:

El sindrome de Apneas Hipopneas Obstructivas del sueño (SAHOS) se define por la presencia de episodios recurrentes de apneas o hipopneas secundarios al colapso de la faringe durante el sueño, que generan desaturaciones y microdespertares. Estos eventos producen respuestas inflamatorias, cardiovasculares, neurocognitivas y metabólicas, que incrementan la morbimortalidad de los pacientes1. La prevalencia de SAHOS está en aumento, debido en parte al incremento de la obesidad dentro de la población en general. Esto hace que el número de consultas y estudios polisomnográficos solicitados aumenten generando largos tiempos de espera y retrasos diagnósticos. La evidencia disponible muestra que la poligrafía respiratoria realizada en el domicilio del paciente es comparable a la polisomnografía realizada en el laboratorio de sueño para el diagnóstico del SAHOS del adulto en pacientes con sospecha clínica.

Qué aporta este trabajo:

Mejorar el conocimiento de los síntomas, comorbilidades y antropometría de los pacientes que son evaluados clínicamente y sometidos a estudios de sueño puede ayudar a optimizar el uso de los recursos médicos y el modelo de historia clínica.

1- Hospital Enrique Tornú

2- Instituto de Investigaciones Médicas Alfredo Lanari

3- E-mail de contacto: rhrojasmd@gmail.com

Recibido: 2019-09-30 Aceptado: 2020-08-11

DOI: http://dx.doi.org/10.31053/1853.0605.v78.n3.25531

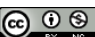

https://creativecommons.org/licenses/by-nc/4.0/

๑) Universidad Nacional de Córdoba

\section{Resumen:}

Introducción: El sindrome de Apneas Hipopneas Obstructivas del sueño (SAHOS) es una patología con una prevalencia en aumento que produce un impacto significativo en la calidad de vida y en la morbimortalidad, fundamentalmente cardiovascular.

Objetivos: Describir síntomas, comorbilidades y medidas antropométricas de pacientes evaluados clínicamente y sometidos a estudios de sueño para identificar variables que se asocian a un Índice de Perturbación Respiratoria (IPR) mayor o igual a 15.

Material y métodos: Se revisaron cuestionarios autoadministrados, antropometría, comorbilidades y estudios de sueño de los pacientes que consultaron por primera vez en el consultorio de patología respiratoria del sueño entre junio de 2012 y mayo de 2016.

Resultados: Entre los 366 pacientes incluidos se encontró $47.5 \%$ con IPR $>30,21,9 \%$ con IPR 15-29.9, 22,1\% con IPR 5-14.9 y 8.5\% con IPR<5. El modelo multivariado usando el IPR $\geq 15$ como variable dependiente demostró que una frecuencia de ronquido mayor a 3 noches por semana (OR 2.89, 95\%IC 1.66 - 5.05), el IMC $\geq 35 \mathrm{Kg} / \mathrm{m} 2$ (OR $2.53,95 \%$ IC $1.35-4.72$ ), apneas presenciadas todas o casi todas las noches (OR 1.95, $95 \%$ IC $1.09-3.49$ ), el sexo masculino (OR $1.81,95 \%$ IC $1.10-2.97$ ) y la presencia de hipertensión arterial (OR 1.67, 95\%IC 1.02 - 2.74) fueron los factores clínicos más significativos.

Conclusiones: En nuestra muestra, los predictores más significativos de la presencia de un IPR $\geq 15$ fueron: una frecuencia de ronquido mayor a 3 noches por semana, el IMC $\geq 35$ $\mathrm{Kg} / \mathrm{m} 2$, apneas presenciadas todas o casi todas las noches, el sexo masculino y la presencia de hipertensión.

Palabras clave: apnea obstructiva del sueño; signos y síntomas; análisis multivariante

\section{Abstract:}

Introduction: Obstructive Sleep Apnea Syndrome is a disease with a growing prevalence worldwide that significantly affects quality of life and increases cardiovascular morbidity and mortality.

Objectives: To describe symptoms, comorbidities and anthropometry of patients who were clinically evaluated and underwent sleep studies to identify variables associated with a Respiratory Disturbance Index (RDI) equal or greater than 15 .

Material and methods: We reviewed data from a self-administered questionary, anthropometry, comorbidities and sleep studies of patients who consulted for the first time at a sleep medicine practice from June 2012 through May 2016.

Results: Among 366 patients included we found $47.5 \%$ with a RDI>30, $21.9 \%$ with a RDI $15-29.9,22.1 \%$ with a RDI $5-14.9$ and $8.5 \%$ with a RDI<5. A multivariate model was built using $\mathrm{RDI} \geq 15$ as the dependent variable. It showed that snoring more than 3 nights per week (OR 2.89, 95\% Cl $1.66-5.05$ ), $\mathrm{BMI} \geq 35 \mathrm{Kg} / \mathrm{m} 2$ (OR 2.53, 95\% Cl $1.35-4.72$ ), witnessed apneas almost every night or every night (OR $1.95,95 \% \mathrm{Cl} 1.09-3.49$ ), male sex $(\mathrm{OR} 1.81,95 \% \mathrm{Cl} 1.10-2.97)$ and the presence of high blood pressure (OR 1.67, $95 \% \mathrm{Cl} 1.02-2.74$ ) were the most significant clinical factors.

Conclusions: In our sample, the most significant predictors of a RDI >15 were: snoring more than 3 nights per week, $\mathrm{BMI} \geq 35 \mathrm{Kg} / \mathrm{m} 2$, witnessed apneas almost every night or every night, male sex and the presence of high blood pressure.

Keywords: sleep apnea, obstructive; signs and symptoms; multivariate analysis

Resumo:

Introdução: A síndrome da Apneia/Hipopneia Obstrutiva do Sono (SAHOS) é uma patologia com prevalência crescente que produz um impacto significativo na qualidade de vida e na morbimortalidade, principalmente cardiovascular.

Objetivos: Descrever sintomas, comorbidades e medidas antropométricas de pacientes avaliados clinicamente e submetidos a estudos do sono para identificar variáveis associadas a um Índice de Distúrbios Respiratórios (IDR) maior ou igual a 15.

Material e métodos: Foram revisados questionários autoadministrados, antropometria, comorbidades e estudos do sono de pacientes consultados no consultório de patologia respiratória do sono entre junho de 2012 e maio de 2016.

Resultados: Entre os 366 pacientes incluídos, $47,5 \%$ foram encontrados com IDR> 30 , $21,9 \%$ com IDR 15 a $29,9,22,1 \%$ com IDR 5 a 14,9 e $8,5 \%$ com IDR $<5$. O modelo multivariado, utilizando a IDR $\geq 15$ como variável dependente, mostrou que uma frequência de ronco superior a 3 noites por semana (OR 2,89, IC 95\% 1,66 - 5,05), IMC $\geq 35 \mathrm{Kg} / \mathrm{m} 2$ (OR 2,53, IC 95\% 1,35 - 4,72), apnéias testemunharam quase todas as noites (OR 1,95, IC 95\% 1,09-3,49), sexo masculino (OR 1,81, IC 95\% 1,10-2,97) e presença de hipertensão arterial (OR 1,67, 95\% IC 1,02 - 2,74) foram os fatores clínicos mais significativos.

Conclusões: Em nossa amostra, os preditores mais significativos da presença de um IPR $\geq 15$ foram: frequência de ronco superior a 3 noites por semana, IMC $\geq 35 \mathrm{Kg} / \mathrm{m} 2$, apnéia testemunhada durante quase todas as noites, sexo masculino e presença de hipertensão.

Palavras-chave: apneia obstrutiva do sono; signos e sintomas; análise multivariada 


\section{Introducción}

Las apneas obstructivas del sueño representan el trastorno respiratorio del sueño más frecuente en la población general. Es una enfermedad crónica de alta relevancia debido a los efectos que padecen los pacientes y su impacto en la salud pública. Los síntomas fundamentales durante el sueño son los ronquidos, las apneas observadas y despertares por asfixia. Durante el día los pacientes presentan somnolencia, sueño no reparador, cansancio y trastornos neurocognitivos. Las apneas obstructivas del sueño se asocian a comorbilidad cardiovascular, trastornos metabólicos y riesgo elevado de accidentes. La prevalencia de SAHOS en la población general aceptada tradicionalmente es de 3.1 a $7.5 \%$ en hombres y 1.2 a $4.5 \%$ en mujeres pre-menopaúsicas, mientras que en la post-menopausia la prevalencia se equipara con el hombre ${ }^{1}$.

El conocimiento de las características clínicas, variables antropométricas y comorbilidades permite identificar con mayor facilidad a los pacientes con alto riesgo de tener SAHOS. La sospecha clínica debe ser confirmada mediante mediciones objetivas para definir el tratamiento más adecuado. La aplicación de presión positiva en vía aérea (CPAP) es el tratamiento de elección en pacientes con SAHOS moderado a severo (IPR $\geq 15$ eventos/hora). El sistema público de salud en Argentina permite acceso a un alto nivel de procedimientos diagnósticos y tratamientos a población económicamente vulnerable. Sin embargo, los recursos públicos no son ilimitados. El objetivo del presente trabajo es describir síntomas, comorbilidades y antropometría de los pacientes que fueron evaluados clínicamente y fueron sometidos a estudios de sueño. Además de identificar variables asociadas a un IPR mayor o igual a 15 que implica requerimiento de tratamiento con CPAP.

\section{Material y Métodos}

Se revisaron los datos de 467 pacientes que consultaron por primera vez entre 1 de junio de 2012 y 31 de mayo de 2016 en el consultorio de sueño del Hospital Tornú (Buenos Aires, Argentina). Tratandose de un centro de referencia de tercer nivel, se reciben pacientes con sospecha clínica de SAHOS referidos desde la atención primaria y otras especialidades. Antes de la consulta, lo pacientes completan un cuestionario autoadministrado en el que se consignan datos personales, escala de somnolencia de Epworth ${ }^{2}$ y 13 preguntas sobre sintomatología de SAHOS basadas en el Consenso Argentino de Trastornos Respiratorios del Sueño ${ }^{3}$. Las preguntas son sobre presencia, frecuencia e intensidad del ronquido, despertares por ahogo o por el ronquido, apneas presenciadas o respiración dificultosa durante el sueño, movimientos bruscos o patadas durante el sueño, cansancio diurno, somnolencia diurna, quedarse dormido manejando, cefalea matinal, dormirse contra su voluntad o en actividades, dificultades en la memoria, concentración o atención.

Durante la consulta se registran datos sobre comorbilidades, tabaquismo y se toman medidas antropométricas. En base a los datos obtenidos se programan estudios simplificados en aquellos pacientes de alto riesgo de disturbios respiratorios relacionados al sueño (DRRS) y polisomnografía en los demás. Los estudios de poligrafía se realizan con dos equipos diferentes. El primero es un equipo Resmed modelo Apnea Link® con registro de señales de flujo aéreo por cánula de presión nasal, ronquido derivado de cánula nasal, oximetría y frecuencia de pulso. El análisis de señales se realiza con el software ApneaLink versión 8 con análisis automático y posterior revisión manual. El segundo equipo es Embla modelo Embletta Gold $\circledast$ con registro de señales de flujo aéreo por cánula de presión nasal, ronquido derivado de cánula nasal, movimiento torácico y abdominal por bandas XactTrace $\AA$ RIP, oximetría de pulso y posición corporal. Las señales se evaluan con el software RemLogic-E versión 1.3 con análisis automático de eventos y revisión manual de los mismos. Para los estudios de polisomnografía se utiliza un equipo ATI Praxis18 AMP18P Lermed S.R.L. En cada estudio se registraron tres canales de EEG: C3, C4 y 01 con referenciales en mastoides (A1 y A2), Dos canales de EOG (derecho e izquierdo), tres canales de EMG, ECG, flujo aéreo por termistor y por cánula de presión nasal, bandas piezoeléctricas torácicas y abdominales para esfuerzo, sensor de posición corporal, oximetría de pulso y micrófono. Los registros se analizan manualmente con el software DelphosDB versión 1.75.32.4 (Lermed S.R.L.) acorde a los estándares de la American Association of Sleep Medicine (AASM) ${ }^{4}$. Los pacientes que no completaron estudios se excluyeron del análisis.

\section{Análisis estadístico}

Se clasificaron a los pacientes según el índice de perturbación respiratorio (IPR) en 4 grupos: IPR $<5$ ( $n=31$ pacientes), IPR $5-$ 14.9 ( $n=81$ pacientes), IPR $15-29.9$ ( $n=80$ pacientes) e IPR $>30$ ( $n=174$ pacientes). La distribución por sexo, antecedentes y comorbilidades se compararon usando el test $\mathrm{chi}^{2}$ de Pearson. Las diferencias entre edad, IMC, circunferencia de cuello, medias y medianas de Epworth se compararon usando el test de KruskalWallis. En variables numéricas, la sensibilidad, especificidad y curva Característica Operativa del Receptor (ROC) se utilizaron para encontrar los mejores puntos de corte para detectar un IPR $\geq 15$. Se calculó el Odds-Ratio por regresión logística comparando dos grupos, pacientes con IPR $\geq 15$ y aquellos con IPR $<15$. Se contruyó un modelo multivariado usando regresión logística múltiple paso a paso. Las variables se incluyeron si $p<0.20$ en el análisis individual. La discriminación del modelo fue evaluada por curvas ROC y Goodnes of fit por el test de Hosmer y Lemershow. El valor de $p$ se consideró significativo con un valor menor a 0.05 .

\section{Resultados}

Se analizaron datos clínicos y respuestas al cuestionario de 366 pacientes que completaron estudios diagnósticos y se encontró un $47.5 \%$ de pacientes con IPR $>30,21.9 \%$ con IPR de 15 a 29.9 , $22.1 \%$ con IPR de 5 a 14.9 y $8.5 \%$ sin trastorno respiratorio del sueño con un IPR<5 (Tabla 1 y 2 ). 
Tabla N¹: Características de los pacientes

\begin{tabular}{|c|c|c|c|c|c|c|}
\hline & $\begin{array}{c}\text { Total } \\
(n=366)\end{array}$ & $\begin{array}{c}\text { IPR }<5 \\
(n=31)\end{array}$ & $\begin{array}{l}\text { IPR 5-14.9 } \\
\quad(n=81)\end{array}$ & $\begin{array}{l}\text { IPR 15-30 } \\
\quad(n=80)\end{array}$ & $\begin{array}{l}\text { IPR }>30 \\
(n=174)\end{array}$ & $\begin{array}{c}\text { Valor de } \\
\text { P }\end{array}$ \\
\hline \multicolumn{7}{|l|}{ Datos demográficos } \\
\hline Sexo masculino & $209(57.1 \%)$ & $14(45.2 \%)$ & $36(44.4 \%)$ & $42(52.5 \%)$ & $117(67.2 \%)$ & 0.002 \\
\hline Sexo femenino & $157(42.9 \%)$ & $17(54.8 \%)$ & $45(55.6 \%)$ & $38(47.5 \%)$ & $57(32.8 \%)$ & \\
\hline Edad & $54.2 \pm 13.6$ & $51.2 \pm 14.3$ & $54.4 \pm 14.1$ & $55.1 \pm 12.2$ & $54.3 \pm 13.9$ & 0.63 \\
\hline \multicolumn{7}{|l|}{ Datos antropométricos } \\
\hline $\mathrm{IMC}$ & $33.1 \pm 7.8$ & $27.3 \pm 4.3$ & $31 \pm 7.2$ & $32.8 \pm 7.5$ & $35.2 \pm 8$ & 0.0001 \\
\hline $\begin{array}{l}\text { Circunferencia cuello (sexo } \\
\text { femenino)* }\end{array}$ & $38.4 \pm 4.1$ & $36.7 \pm 5.1$ & $37.6 \pm 3.7$ & $37.9 \pm 3.8$ & $39.8 \pm 4.2$ & 0.004 \\
\hline $\begin{array}{l}\text { Circunferencia cuello (sexo } \\
\text { masculino)* }\end{array}$ & $43.9 \pm 4.5$ & $40.2 \pm 4$ & $41.8 \pm 4.4$ & $44.7 \pm 4.2$ & $44.6 \pm 4.3$ & 0.0004 \\
\hline \multicolumn{7}{|l|}{ Somnolencia } \\
\hline Epworth (mediana) & $10.5(16-5)$ & $6(10-3)$ & $11(15-5)$ & $13(17-7)$ & $11(18-5)$ & 0.001 \\
\hline \multicolumn{7}{|l|}{ Antecedentes y comorbilidades } \\
\hline Tabaquismo & $151(41.8 \%)$ & $10(32.3 \%)$ & $32(39.5 \%)$ & $25(31.3 \%)$ & $84(48.3 \%)$ & 0.046 \\
\hline $\begin{array}{l}\text { Menopausia } \\
\text { (femenino) }\end{array}$ & $116(73.9 \%)$ & $10(58.8 \%)$ & $31(68.9 \%)$ & $30(78.9 \%)$ & $45(78.9 \%)$ & 0.27 \\
\hline Hipercolesterolemia & $152(41.5 \%)$ & $11(35.5 \%)$ & $33(40.7 \%)$ & $28(35 \%)$ & $80(45.9 \%)$ & 0.35 \\
\hline Diabetes & $64(17.5 \%)$ & 0 & $12(14.8 \%)$ & $13(16.3 \%)$ & $39(22.4 \%)$ & 0.019 \\
\hline Coronariopatía & $33(9 \%)$ & 0 & $8(9.8 \%)$ & $8(10 \%)$ & $17(9.8 \%)$ & 0.339 \\
\hline Arritmias & $43(11.7 \%)$ & $1(3.23 \%)$ & $11(13.6 \%)$ & $12(15 \%)$ & $19(10.9 \%)$ & 0.339 \\
\hline $\mathrm{ACV}$ & $21(5.7 \%)$ & $1(3.23 \%)$ & $5(6.17 \%)$ & $6(7.5 \%)$ & $9(5.17 \%)$ & 0.813 \\
\hline Hipotiroidismo & $53(14.5 \%)$ & 0 & $15(18.5 \%)$ & $14(17.5 \%)$ & $24(13.8 \%)$ & 0.073 \\
\hline HTA & $192(52.5 \%)$ & $9(29 \%)$ & $39(48.2 \%)$ & $44(55 \%)$ & $100(57.5 \%)$ & 0.025 \\
\hline
\end{tabular}


Tabla $N^{\circ} 2$ : Respuestas al cuestionario autoadministrado en los diferentes grupos

\begin{tabular}{|c|c|c|c|c|c|}
\hline & & $\begin{array}{c}\text { IPR }<5 \\
(n=31)\end{array}$ & $\begin{array}{c}\text { IPR 5-15 } \\
(n=81)\end{array}$ & $\begin{array}{c}\text { IPR 15-30 } \\
(n=80)\end{array}$ & $\begin{array}{c}\text { IPR }>30 \\
(n=174)\end{array}$ \\
\hline 1. & Alguien le dijo alguna vez que ronca? & $25(80.6 \%)$ & $73(90.1 \%)$ & $75(94.9 \%)$ & $168(96.5 \%)$ \\
\hline \multirow[t]{5}{*}{2.} & De acuerdo a lo que le dijeron, mencione con qué frecuencia ronca. & & & & \\
\hline & Rara vez (unas pocas veces en su vida) & $4(12.9 \%)$ & $3(3.7 \%)$ & $3(3.7 \%)$ & $2(1.1 \%)$ \\
\hline & Algunas veces (varias noches al mes) & $10(32.3 \%)$ & $16(19.7 \%)$ & $10(12.5 \%)$ & $12(6.9 \%)$ \\
\hline & De 3 a 5 noches por semana. & $0(0 \%)$ & $10(12.3 \%)$ & $8(10 \%)$ & $12(6.9 \%)$ \\
\hline & Todas o casi todas las noches. & $10(32.3 \%)$ & $42(51.8 \%)$ & $54(67.5 \%)$ & $141(81 \%)$ \\
\hline \multirow[t]{5}{*}{3.} & Que intensidad tiene su ronquido (según comentarios). & & & & \\
\hline & Un poco más intenso que la respiración profunda. & $9(29 \%)$ & $17(21 \%)$ & $15(18.7 \%)$ & $19(10.9 \%)$ \\
\hline & Igual que un gruñido o que la voz al hablar. & $3(9.7 \%)$ & $14(17.3 \%)$ & $11(13.7 \%)$ & $17(9.8 \%)$ \\
\hline & Más alto que la voz. & $6(19.3 \%)$ & $13(16 \%)$ & $15(18.7 \%)$ & $34(19.5 \%)$ \\
\hline & Muy alto, se escucha con la puerta cerrada. & $6(19.3 \%)$ & $25(30.9 \%)$ & $31(38.7 \%)$ & $91(52.3 \%)$ \\
\hline 4. & Se ha despertado con sensación de ahogo o por el sonido de sus propios ronquidos? & $14(45.2 \%)$ & $58(71.6 \%)$ & $57(71.2 \%)$ & $123(70.7 \%)$ \\
\hline \multirow[t]{6}{*}{5.} & Según lo que le han dicho, con qué frecuencia ha dejado de respirar o h & lo con dificul & tras dormía & & \\
\hline & Nunca & $17(54.8 \%)$ & $27(33.3 \%)$ & $21(26.2 \%)$ & $22(12.6 \%)$ \\
\hline & Rara vez (solo contadas veces) & $6(19.3 \%)$ & $11(13.6 \%)$ & $4(5 \%)$ & $14(8 \%)$ \\
\hline & Algunas veces (algunas noches al mes) & $5(16.1 \%)$ & $11(13.6 \%)$ & $9(11.2 \%)$ & $26(14.9 \%)$ \\
\hline & Frecuentemente (por lo menos 1 vez a la semana, aunque no siempre) & $2(6.4 \%)$ & $11(13.6 \%)$ & $13(16.2 \%)$ & $27(15.5 \%)$ \\
\hline & Muy frecuentemente (todas o casi todas las noches) & $1(3.2 \%)$ & $21(25.9 \%)$ & $33(41.2 \%)$ & $85(48.8 \%)$ \\
\hline 6. & Patea o tiene movimientos bruscos o anormales durante el sueño? & $14(45.2 \%)$ & $47(58 \%)$ & $38(47.5 \%)$ & $86(49.4 \%)$ \\
\hline 7. & Siente usted excesivo cansancio durante el día? & $22(71 \%)$ & $62(76.5 \%)$ & $64(80 \%)$ & $141(81 \%)$ \\
\hline \multirow[t]{5}{*}{8.} & Siente usted excesivo sueño durante el día? & & & & \\
\hline & Rara vez (solo contadas veces) & $9(29 \%)$ & $18(22.2 \%)$ & $19(23.7 \%)$ & $28(16.1 \%)$ \\
\hline & Algunas veces (algunos días al mes) & $9(29 \%)$ & $16(19.7 \%)$ & $9(11.2 \%)$ & $33(19 \%)$ \\
\hline & Frecuentemente (por lo menos 1 vez a la semana, aunque no siempre) & $3(9.7 \%)$ & $14(17.3 \%)$ & $12(15 \%)$ & $35(20.1 \%)$ \\
\hline & Muy frecuentemente (todos o casi todas los días) & $7(22.6 \%)$ & $32(39.5 \%)$ & $38(47.5 \%)$ & $76(43.7 \%)$ \\
\hline \multirow[t]{4}{*}{9.} & Se quedó alguna vez dormido mientras manejaba? & & & & \\
\hline & $\mathrm{Si}$ & $4(44.4 \%)$ & $5(17.9 \%)$ & $11(39.3 \%)$ & $34(43 \%)$ \\
\hline & No & $5(55.6 \%)$ & $23(82.1 \%)$ & $17(60.7 \%)$ & $45(57 \%)$ \\
\hline & No conduce & $22(71 \%)$ & $53(65.4 \%)$ & $52(65 \%)$ & $95(54.6 \%)$ \\
\hline 10. & Se despierta por la mañana con dolor de cabeza? & $14(45.2 \%)$ & $40(49.4 \%)$ & $43(53.7 \%)$ & $71(40.8 \%)$ \\
\hline \multirow{6}{*}{11.} & Se queda usted dormido contra su voluntad, o debe luchar para no dor & & & & \\
\hline & Nunca & $14(45.2 \%)$ & $23(28.4 \%)$ & $25(31.2 \%)$ & $43(24.7 \%)$ \\
\hline & Rara vez (solo contadas veces) & $6(19.3 \%)$ & $20(24.7 \%)$ & $9(11.2 \%)$ & $25(14.4 \%)$ \\
\hline & Algunas veces (algunas veces al mes) & $4(12.9 \%)$ & $8(9.9 \%)$ & $12(15 \%)$ & $23(13.2 \%)$ \\
\hline & Frecuentemente (por lo menos 1 vez a la semana, aunque no siempre) & $3(9.7 \%)$ & $11(13.6 \%)$ & $11(13.7 \%)$ & $22(12.6 \%)$ \\
\hline & Muy frecuentemente (todas o casi todas los días) & $4(12.9 \%)$ & $19(23.5 \%)$ & $23(28.7 \%)$ & $61(35.1 \%)$ \\
\hline \multirow{6}{*}{12.} & Se queda dormido durante actividades habituales o en el trabajo? & & & & \\
\hline & Nunca & $22(71 \%)$ & $42(51.8 \%)$ & $43(53.7 \%)$ & $77(44.2 \%)$ \\
\hline & Rara vez (solo contadas veces) & $2(6.4 \%)$ & $11(13.6 \%)$ & $11(13.7 \%)$ & $28(16.1 \%)$ \\
\hline & Algunas veces (algunas veces al mes) & $4(12.9 \%)$ & $12(14.8 \%)$ & $7(8.7 \%)$ & $18(10.4 \%)$ \\
\hline & Frecuentemente (por lo menos 1 vez a la semana, aunque no siempre) & $1(3.2 \%)$ & $4(4.9 \%)$ & $7(8.7 \%)$ & $16(9.2 \%)$ \\
\hline & Muy frecuentemente (todas o casi todas los días) & $2(6.4 \%)$ & $12(15 \%)$ & $12(15 \%)$ & $35(20.1 \%)$ \\
\hline \multirow[t]{4}{*}{13.} & En el último año notó usted que & & & & \\
\hline & Tiene dificultad para concentrarse? & $18(58.1 \%)$ & $54(66.6 \%)$ & $45(56.2 \%)$ & $83(47.7 \%)$ \\
\hline & Ha disminuido su memoria? & $22(71 \%)$ & $60(74.1 \%)$ & $59(73.7 \%)$ & $95(54.6 \%)$ \\
\hline & Le cuesta más esfuerzo mantener la atención? & $17(54.8 \%)$ & $54(66.6 \%)$ & $51(63.7 \%)$ & $87(50 \%)$ \\
\hline
\end{tabular}

La distribución por sexo fue similar en todos los grupos, excepto en los pacientes con IPR > 30, en los que la relación hombre mujer fue de 2.05:1. La edad media fue similar en todos los grupos. EI IMC y la circunferencia de cuello se asociaron significativamente a la severidad del SAHOS. La escala de somnolencia de Epworth mostró diferencias significativas entre los grupos, especialmente en comparación con los pacientes sin trastorno respiratorio del sueño y los demás. Entre los antecedentes y comorbilidades se encontraron diferencias significativas solo en HTA, diabetes y tabaquismo.
Para identificar variables potenciales para ser incluidas en el modelo multivariado, se realizó un análisis individual usando el IPR $\geq 15$ como variable dependiente (Tabla 3). Las variables numéricas se categorizaron para mejor aplicación clínica. Se utilizaron curvas ROC para evaluar si la circunferencia de cuello discrimina sujetos con IPR $\geq 15$ en ambos sexos. El punto de corte en mujeres fue de $37 \mathrm{~cm}$ con una sensibilidad de $71.1 \%$ y una especificidad de $53.6 \%$. El punto de corte en hombres fue de $43 \mathrm{~cm}$. con una sensibilidad de $72.2 \%$ y una especificidad de $63 \%$. 
Tabla $\mathrm{N}^{\circ}$ : Análisis individual de variables potenciales a incluir en el modelo multivariado

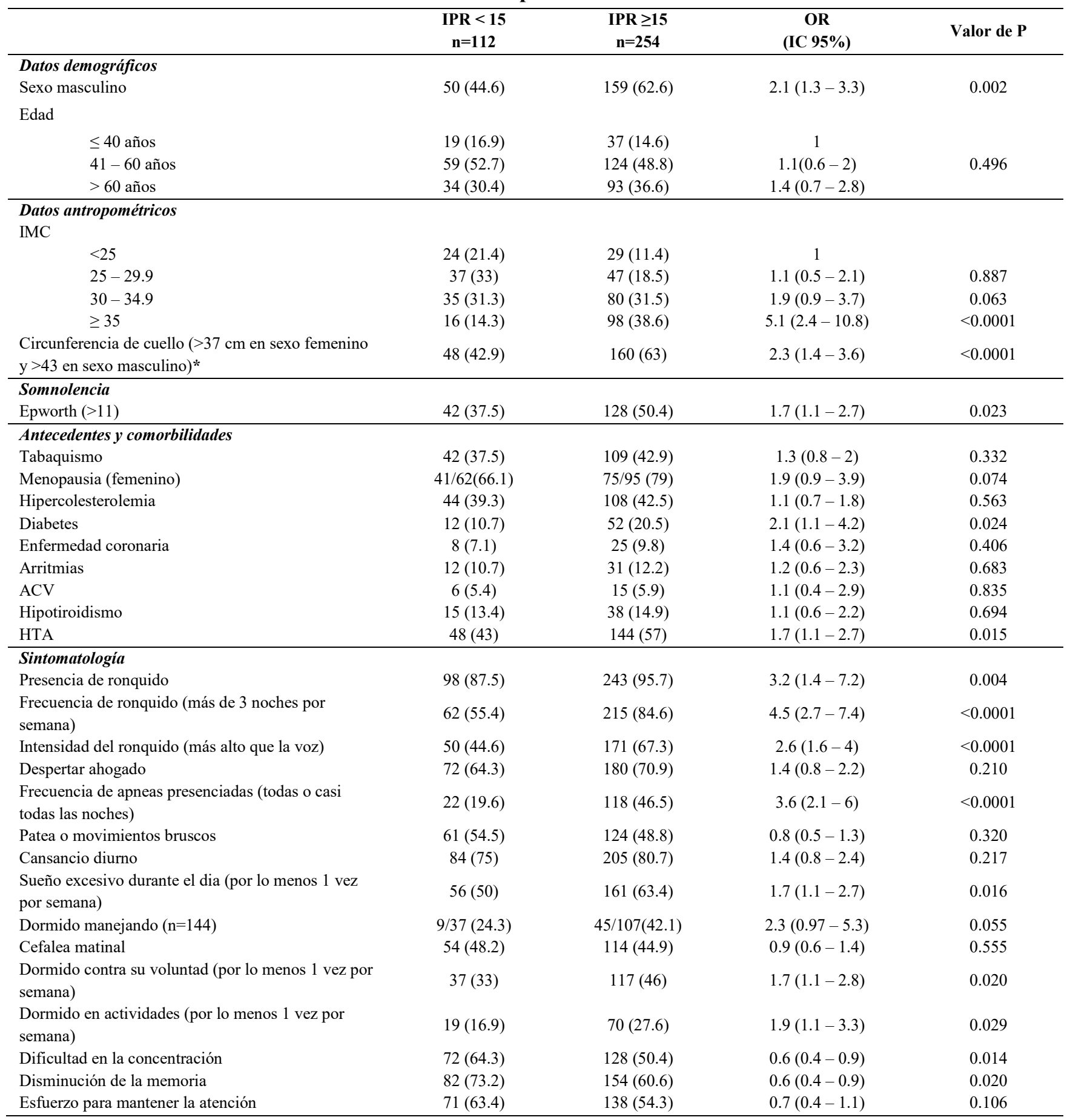

A fin de identificar los mejores predictores de un IPR $\geq 15$, se construyó un modelo multivariado usando regresión logística múltiple. (Tabla 4) Las probabilidades de un IPR $\geq 15$ predichas por el modelo fue del $27,6 \%$ cuando ninguna de las variables está presente, $57.2 \%$ cuando una o dos están presentes y $94.25 \%$ cuando todas son positivas.

Tabla $\mathbf{N}^{\circ}$ 4: Modelo Multivariado. Factores de riesgo que correlacionan con un IPR $\geq 15$

\begin{tabular}{lcc}
\hline Variables & OR (95\%IC) & p \\
\hline Frecuencia de ronquido (más de 3 veces por semana) & $2.89(1.66-5.05)$ & $<0.0001$ \\
IMC $\geq 35$ & $2.53(1.35-4.72)$ & 0.004 \\
Frecuencia de apneas presenciadas (todas o casi todas las noches) & $1.95(1.09-3.49)$ & 0.025 \\
Sexo masculino & $1.81(1.10-2.97)$ & 0.020 \\
Hipertensión Arterial & $1.67(1.02-2.74)$ & 0.040 \\
\hline Goodness of fit p0.2872
\end{tabular}

Goodness of fit $\mathrm{p} 0.2872$

ROC: 0.75 


\section{Discusión}

Se analizaron datos de la dinámica de trabajo usada a diario en nuestro consultorio de sueño. Consiste en tres pasos: el primero es un cuestionario estructurado autoreportado que incluye la escala de somnolencia de Epworth. El segundo paso se realiza durante la consulta e incluye mediciones antropométricas y detección de comorbilidades. El tercer paso es la realización de un estudio de sueño, la elección del tipo de estudio se realiza acorde a los datos obtenidos en los primeros dos pasos.

En nuestro estudio, los predictores más significativos para la presencia de un IPR mayor a 15 en el modelo multivariado fueron: ronquido más de 3 noches por semana, IMC mayor o igual a 35 $\mathrm{Kg} / \mathrm{m} 2$, apneas presenciadas todas o casi todas las noches, sexo masculino y la presencia de HTA. A pesar de que el objetivo del trabajo no es la creación de una herramienta de screening para la población general, las variables encontradas son similares a las de los reportes internacionales. El cuestionario de Berlin, una herramienta desarrollada para la atención primaria, usa preguntas similares a las de nuestro estudio, con la diferencia de tener un corte del IMC de $30 \mathrm{Kg} / \mathrm{m} 2$ y no usar el sexo en la puntuación final ${ }^{5}$. El cuestionario STOP-BANG incluye HTA, IMC y sexo de la misma forma que nuestros hallazgos, con la diferencia en que nuestro estudio incluye la frecuencia de apneas presenciadas. Este cuestionario se diferencia con el nuestro en que se diseñó y validó en paciente quirúrgicos durante el preoperatorio ${ }^{6}$. Schiavone y col. estudiaron la precisión diagnóstica de los parámetros clínicos para detectar SAHOS $(\mathrm{IAH}>15)$ con poligrafía respiratoria en pacientes con HTA y encontraron un AUC-ROC de 0.66 con un STOP-BANG de más de 3 y un AUC-ROC de 0.9 con un STOP-BANG de más de 57 . El AUC-ROC de nuestro modelo fue de 0.75 . Sin embargo, no podemos comparar directamente ambos estudios debido a que la muestra de dicho estudio estaba compuesta por pacientes con una conocida comorbilidad (HTA) y nuestra muestra fueron pacientes que consultaron por síntomas de SAHOS o fueron referidos por otros médicos con sospecha de SAHOS.

A pesar de ser algo subjetivo, el ronquido fue el síntoma más reportado y se observó en más del $90 \%$ de los pacientes con SAHOS de la muestra. Es también una causa frecuente de derivación y consulta en los pacientes, especialmente cuando interrumpe el sueño de la pareja. La frecuencia de ronquido fue el predictor que presentó mayor asociación con un IPR mayor o igual a 15. Estudios previos encontraron que la frecuencia y la intensidad del ronquido son predictores de severidad. En una evaluación objetiva (medición de intensidad en decibeles) de 1643 pacientes, Nimrod y col. encontraron una correlación positiva entre la intensidad y la severidad del SAHOS $^{8}$. En nuestro estudio, la intensidad del ronquido se asoció con la presencia de un IPR mayor o igual a 15. Sin embargo, no alcanzó significación estadística en el modelo multivariado.

La asociación entre el IMC y la severidad del SAHOS fue estadísticamente significativa. El IMC es el factor de riesgo antropométrico más significativo y representa otra razón frecuente para el pedido de estudios de sueño. En un estudio sistemático de pacientes en plan de cirugía bariátrica con un IMC mayor a 40, aún en ausencia de síntomas, se demostró la presencia de SAHOS en $70 \%$ de los casos ${ }^{9}$.

La referencia de la pareja en cuanto a la frecuencia de dificultades respiratorias 0 apneas presenciadas mostró diferencias estadísticamente significativas entre los grupos acorde al IPR. Este resultado es consistente con la literatura que describe que el $49 \%$ de los pacientes con un IPR mayor a 15 tenían una frecuencia autoreportada de dificultades respiratorias durante la noche de más de 3 veces por semana ${ }^{4}$.

En concordancia con reportes previos, el sexo masculino fue un factor de riesgo. La prevalencia de SAHOS en la población general es cercana al $15 \%$ en hombres y $5 \%$ en mujeres entre 30 y 70 años considerando un IPR mayor o igual a 15 o un IPR mayor a 5 con presencia de somnolencia ${ }^{10}$.
En nuestra muestra, la HTA fue claramente la comorbilidad con mayor fuerza de asociación con SAHOS. En algunos estudios la asociación entre SAHOS severo e HTA alcanza hasta el $45 \%$ en pacientes con diagnóstico previo de hipertensión ${ }^{11}$. La presencia de un IPR mayor a 15 se asocia en un $30 \%$ a la HTA y en más del $80 \%$ a la HTA resistente a las drogas ${ }^{12}$. Comparativamente con los pacientes con hipertensión controlada por la medicación, los resistentes al tratamiento antihipertensivo, tienen mayor desplazamiento de volumen rostral de líquidos desde miembros inferiores, fenómeno que explicaría el empeoramiento del IPR ${ }^{13}$. Sin embargo, en grandes estudios poblacionales, la asociación deja de ser significativa cuando se ajusta con otras variables de riesgo para presentar hipertensión. En el American Sleep Heart Health Study (ASHHS) en el ajuste por IMC, el IPR deja de ser predictor de HTA. En el Vitoria Sleep Cohort, el IPR como factor de riesgo para desarrollar HTA deja de ser significativo cuando se ajusta por sexo, edad, circunferencia del cuello, nivel de actividad física y consumo de sustancias como alcohol, café y tabaquismo ${ }^{14,15}$.

Otras variables que se asociaron con IPR mayor o igual a 15 en el análisis individual fueron la circunferencia de cuello, diabetes, tabaquismo, somnolencia diurna excesiva, intensidad del ronquido, dificultades en la concentración y la memoria. Sin embargo, no alcanzaron significación estadística en el modelo multivariado.

Se encontraron diferentes puntos de corte para circunferencia del cuello en la literatura, por lo que se eligió un valor estadístico adecuado para la muestra estudiada. En mujeres, el punto de corte elegido de $37 \mathrm{~cm}$. fue similar a estudios previos. En hombres fue de $43 \mathrm{~cm}$., más alto que lo previamente reportado ${ }^{16-17}$. El ASHHS mostró que la circunferencia de cuello es el mejor predictor de SAHOS en adultos comparado con otros parámetros clínicos como el ronquido, sexo, edad e incluso, el IMC ${ }^{18}$. Nuestra población tiene una distribución étnica diferente (predominantemente hispana) que podría explicar estas diferencias.

El National Institute of Health reportó que el SAHOS es tan prevalente como la diabetes ${ }^{19}$. La prevalencia de SAHOS entre pacientes diabéticos obesos ha sido estimada en $86 \%$ por Sleep AHEAD (Action for Health in Diabetes) ${ }^{20}$. Además, estudios sugieren que la asociación de SAHOS con glucemia incrementada e insulinoresistencia puede ser independiente de la presencia de obesidad $^{21}$. El mecanismo involucrado podría ser el incremento de actividad simpática que lleva a la degradación del glicógeno y a gluconeogénesis. También existe evidencia que los pacientes con SAHOS y buena adherencia al tratamiento con CPAP reducen la glucemia y los niveles de $\mathrm{HbA1C}$ cuando lo usan más días y horas ${ }^{22}$

Hoffstein lideró un estudio epidemiológico sobre apneas del sueño y tabaquismo en el cual incluyó 3059 pacientes y encontró que los fumadores activos tenían mayor proporción de SAHOS más severo (IPR mayor a 50 eventos/hora) ${ }^{23}$. En contraste, una revisión de la SEPAR concluyó que la asociación es débil, minimizando su importancia en la fisiopatología de la apnea del sueño ${ }^{24}$.

El síntoma somnolencia se refiere al incremento de la propensión a dormitarse, y subjetiva compulsión al sueño, así como la tendencia a tomar siestas involuntarias o sufrir ataques de sueño, cuando el sueño no es deseado. En nuestro estudio, la frecuencia de somnolencia diurna excesiva de más de 1 vez por semana se asoció a un IPR mayor o igual a 15. La escala de somnolencia de Epworth mostró diferencias entre los pacientes sin trastorno respiratorio del sueño y los pacientes con SAHOS. Sin embargo, un problema con esta prueba es su interpretación en diferentes momentos del día y que puede subestimar el síntoma somnolencia cuando el paciente tiene una percepción inadecuada de su condición $^{25}$. Hay pobre correlación entre la escala con mediciones objetivas como el Test de Latencias Múltiples ${ }^{26}$. La escala de somnolencia de Epworth es una apreciación estable cuando se utiliza en sujetos sanos y en aquellos bajo tratamiento con CPAP.

Los trastornos neurocognitivos fueron curiosamente bajos en los pacientes con IPR mayor o igual a 15. Estos resultados contrastan con la literatura que describe déficit de atención y alteraciones en la recolección consciente de la memoria en pacientes con SAHOS severo $^{27}$. Estas diferencias se deben probablemente a la forma en 
que se planteó la pregunta. Una respuesta de si o no es insuficiente para detectar trastornos neurocognitivos adecuadamente, éstos requieren el uso de herramientas específicas. Además, otras comorbilidades pueden ser la razón del mayor número de deterioro cognitivo en los pacientes con IPR menor a 15.

Nuestro estudio tiene muchas limitaciones. Los cuestionarios autoadministrados habitualmente no reflejan la realidad de los pacientes debido a la percepción de los síntomas y el nivel de educación. El uso de poligrafía respiratoria subestima el IPR hasta en un $40 \%$ comparado con la polisomnografía estándar. No logra detectar hipopneas que desencadenan microdespertares sin desaturación y además el índice no es directamente comparable debido al cociente utilizado (tiempo total de registro versus tiempo total de sueño). Aún con estos problemas, el protocolo de manejo con poligrafía en el ámbito clínico no es inferior a la polisomnografía y presenta costos sustancialmente menores ${ }^{28}$.

Otra limitante de nuestro estudio fue que la muestra se compone principalmente de pacientes con sospecha clínica de trastornos respiratorios del sueño y, por lo tanto, no es posible extrapolarla a la población general.

\section{Conclusiones}

En nuestra población, los predictores más significativos de la presencia de un IPR mayor o igual a 15 fueron: una frecuencia de ronquido mayor a 3 noches por semana, el IMC mayor o igual a 35 $\mathrm{Kg} / \mathrm{m} 2$, apneas presenciadas todas o casi todas las noches, el sexo masculino y la presencia de HTA. Estos hallazgos pueden servir en la práctica diaria para optimizar el uso de recursos diagnósticos y mejorar el costo-beneficio de los servicios de salud. El score obtenido de estos predictores será aplicado a futuros análisis de la población de nuestro centro.

Limitaciones de responsabilidad:

La responsabilidad del trabajo es solo de los autores.

Conflicto de interés:

Ninguno.

\section{Fuentes de apoyo:}

La presente investigación no contó con fuentes de financiación.

\section{Originalidad del trabajo:}

Los participantes de este trabajo ceden el derecho de autor a la Universidad Nacional de Córdoba para publicar en la RFCM y realizar las traducciones necesarias al idioma inglés.

\section{Cesión de derechos:}

Autorizamos la publicación del manuscrito por la Revista de la Facultad de Ciencias Médicas y cedemos derechos de publicación.

\section{Bibliografía}

1. Nogueira F, Borsini E, Cambursano H, Smurra M, Dibur E, Franceschini C, Perez-Chada D, Larrateguy L, Nigro C. Guías prácticas de diagnóstico y tratamiento del síndrome de apneas e hipopneas obstructivas del sueño: Actualización 2019. Rev. am. med. $\quad$ Respir. $2019 \quad$ Mar; 19(1):59-90. http://www.scielo.org.ar/scielo.php?script=sci arttext\&pid=S1852$\underline{236 \times 2019000100007}$

2. Johns MW. A new method for measuring daytime sleepiness: the Epworth sleepiness scale. Sleep. 1991 Dec;14(6):540-5. doi: 10.1093/sleep/14.6.540.

3. Sala H, Nigro C, Rabec C, Guardia AS, Smurra M. Consenso Argentino de trastornos respiratorios vinculados al sueño [Argentine consensus on sleep-related respiratory disorders]. Medicina (B Aires). 2001;61(3):351-63. Spanish.
4. Berry R, Brooks R, Gamaldo C, Harding S, Lloyd R, Marcus C, Vaughn B. The AASM Manual for the Scoring of Sleep and Associated Events. Rules, terminology and technical specifications. Version 2.2 American Academy of Sleep Medicine. 2015; 18-78

5. Netzer NC, Stoohs RA, Netzer CM, Clark K, Strohl KP. Using the Berlin Questionnaire to identify patients at risk for the sleep apnea syndrome. Ann Intern Med. 1999 Oct 5;131(7):485-91. doi: 10.7326/0003-4819-131-7-199910050-00002.

6. Chung F, Yang Y, Brown R, Liao P. Alternative scoring models of STOP-bang questionnaire improve specificity to detect undiagnosed obstructive sleep apnea. J Clin Sleep Med. 2014 Sep 15;10(9):951-8. doi: 10.5664/jcsm.4022.

7. Schiavone M, Ernst G, Blanco M, Avaca H, Acosta AL, Nosetto $D$, Manuale O, Salvado A, Borsini E. Performance of questionnaires aimed at detecting sleep disorders in patients attending a hypertension center. Clin Exp Hypertens. 2019;41(7):687-691. doi: 10.1080/10641963.2018.1539095.

8. Young $T$, Shahar E, Nieto FJ, Redline S, Newman AB, Gottlieb DJ, Walsleben JA, Finn L, Enright P, Samet JM; Sleep Heart Health Study Research Group. Predictors of sleep-disordered breathing in community-dwelling adults: the Sleep Heart Health Study. Arch Intern Med. 2002 Apr 22;162(8):893-900. doi: 10.1001/archinte.162.8.893

9. Maimon N, Hanly PJ. Does snoring intensity correlate with the severity of obstructive sleep apnea? J Clin Sleep Med. 2010 Oct 15;6(5):475-8.

10. Jonas $D E$, Amick HR, Feltner $C$, Weber RP, Arvanitis M, Stine A, Lux L, Harris RP. Screening for Obstructive Sleep Apnea in Adults: Evidence Report and Systematic Review for the US Preventive Services Task Force. JAMA. 2017 Jan 24;317(4):415433. doi: 10.1001/jama.2016.19635. Erratum in: JAMA. 2017 Mar 28;317(12):1278.

11. Baguet JP, Lévy P, Barone-Rochette G, Tamisier R, Pierre H, Peeters M, Mallion JM, Pépin JL. Masked hypertension in obstructive sleep apnea syndrome. J Hypertens. 2008 May;26(5):885-92. doi: 10.1097/HJH.0b013e3282f55049.

12. Logan AG, Perlikowski SM, Mente A, Tisler A, Tkacova R, Niroumand $M$, Leung RS, Bradley TD. High prevalence of unrecognized sleep apnoea in drug-resistant hypertension. J Hypertens. 2001 Dec;19(12):2271-7. doi: 10.1097/00004872200112000-00022.

13. Friedman O, Bradley TD, Chan CT, Parkes R, Logan AG. Relationship between overnight rostral fluid shift and obstructive sleep apnea in drug-resistant hypertension. Hypertension. 2010 Dec;56(6):1077-82.

10.1161/HYPERTENSIONAHA.110.154427.

14. O'Connor GT, Caffo B, Newman AB, Quan SF, Rapoport DM, Redline S, Resnick HE, Samet J, Shahar E. Prospective study of sleep-disordered breathing and hypertension: the Sleep Heart Health Study. Am J Respir Crit Care Med. 2009 Jun 15;179(12):1159-64. doi: 10.1164/rccm.200712-18090C.

15. Cano-Pumarega I, Durán-Cantolla J, Aizpuru F, MirandaSerrano E, Rubio R, Martínez-Null C, de Miguel J, Egea C, Cancelo $L$, Alvarez A, Fernández-Bolaños $M$, Barbé $F$. Obstructive sleep apnea and systemic hypertension: Iongitudinal study in the general population: the Vitoria Sleep Cohort. Am J Respir Crit Care Med. 2011 Dec 1;184(11):1299-304. doi: 10.1164/rccm.201101-01300C. 
16. Soylu AC, Levent E, Sarıman N, Yurtlu S, Alparslan S, Saygı A. Obstructive sleep apnea syndrome and anthropometric obesity indexes. Sleep Breath. 2012 Dec;16(4):1151-8. doi: 10.1007/s11325-011-0623-9.

17. Chung $F$, Yegneswaran $B$, Liao $P$, Chung $S A$, Vairavanathan $S$, Islam S, Khajehdehi A, Shapiro CM. STOP questionnaire: a tool to screen patients for obstructive sleep apnea. Anesthesiology. 2008 May;108(5):812-21. doi: 10.1097/ALN.0b013e31816d83e4.

18. Caffo B, Diener-West M, Punjabi NM, Samet J. A novel approach to prediction of mild obstructive sleep disordered breathing in a population-based sample: the Sleep Heart Health Study. Sleep. 2010 Dec;33(12):1641-8. doi: 10.1093/sleep/33.12.1641.

19. Lee W, Nagubadi S, Kryger MH, Mokhlesi B. Epidemiology of Obstructive Sleep Apnea: a Population-based Perspective. Expert Rev Respir Med. 2008;2(3):349-364. doi:10.1586/17476348.2.3.349

20. Foster GD, Sanders MH, Millman R, Zammit G, Borradaile KE, Newman $A B$, Wadden TA, Kelley $D$, Wing RR, Sunyer FX, Darcey $V$, Kuna ST; Sleep AHEAD Research Group. Obstructive sleep apnea among obese patients with type 2 diabetes. Diabetes Care. 2009 Jun;32(6):1017-9. doi: 10.2337/dc08-1776.

21. Ip MS, Lam B, Ng MM, Lam WK, Tsang KW, Lam KS. Obstructive sleep apnea is independently associated with insulin resistance. Am J Respir Crit Care Med. 2002 Mar 1;165(5):670-6. doi: 10.1164/ajrccm.165.5.2103001

22. Dawson A, Abel SL, Loving RT, Dailey G, Shadan FF, Cronin $J W$, Kripke DF, Kline LE. CPAP therapy of obstructive sleep apnea in type 2 diabetics improves glycemic control during sleep. J Clin Sleep Med. 2008 Dec 15;4(6):538-42.

23. Hoflstein V. Relationship between smoking and sleep apnea in clinic population. Sleep. 2002 Aug 1;25(5):519-24.

24. C. Balaguer et al. Tabaco y trastornos del sueño. Arch Bronconeumol. 2009;45(9):449-458

25. Nam H, Lim JS, Kim JS, Lee KJ, Koo DL, Lee C. Sleep Perception in Obstructive Sleep Apnea: A Study Using Polysomnography and the Multiple Sleep Latency Test. J Clin Neurol. 2016 Apr;12(2):230-5. doi: 10.3988/jcn.2016.12.2.230

26. Benbadis SR, Mascha E, Perry MC, Wolgamuth BR, Smolley LA, Dinner DS. Association between the Epworth sleepiness scale and the multiple sleep latency test in a clinical population. Ann Intern Med. 1999 Feb 16;130(4 Pt 1):289-92. doi: 10.7326/0003-4819-1304-199902160-00014.

27. Daurat A, Sarhane M, Tiberge M. Syndrome d'apnées obstructives du sommeil et cognition : une revue [Obstructive sleep apnea syndrome and cognition: A review]. Neurophysiol Clin. 2016 Jun;46(3):201-15. French. doi: 10.1016/j.neucli.2016.04.002.

28. Corral J, Sánchez-Quiroga MÁ, Carmona-Bernal C, SánchezArmengol Á, de la Torre AS, Durán-Cantolla J, Egea CJ, Salord N, Monasterio C, Terán J, Alonso-Alvarez ML, Muñoz-Méndez J, Arias EM, Cabello M, Montserrat JM, De la Peña M, Serrano JC, Barbe F, Masa JF; Spanish Sleep Network. Conventional Polysomnography Is Not Necessary for the Management of Most Patients with Suspected Obstructive Sleep Apnea. Noninferiority, Randomized Controlled Trial. Am J Respir Crit Care Med. 2017 Nov 1;196(9):1181-1190. doi: 10.1164/rccm.201612-2497OC 